\section{High-pressure freezing for cryoelectron microscopy}

\section{Jacques Dubochet}

The inside of a living cell is crowded and extremely mobile at the molecular level ${ }^{1}$. For the moment, we can only imagine, by using the theory of diffusion and our fragmentary knowledge of molecular motors, how the contents of the cell move and interact. The ultimate aim of cell biologists is to capture these movements in real-time but, for now, cryoelectron microscopy helps us to obtain snapshols of 'suspended' or 'frozen' life. These pictures tell us much about the structure of cells, and a new development, highpressure freezing (HPF), promises to extend our knowledge even further.

For electron microscopy (EM), the specimen is held under vacuum. Consequently, the water in a biological sample cannot be kept in its natural state - the specimen must be fixed in some way. One major question is whether or not fixed tissues resemble the living tissue. Numerous fixation processes have been used over the years and have had varying degrees of success. Cryofixation, as a physical technique to preserve cellular ultrastructure, has provided an important alternative to conventional chemical fixation. Cryofixation is rapid, occurring in milliseconds rather than the seconds or minutes needed for chemical fixation. This means that all the cellular components are simulta neously stabilized and there is less time for the morphology of the cell to be altered; therefore, cryofixed tissue is more likely to reflect the native structure of living cells. Additionally, cryofixation is often the method of choice for immunocytochemistry because it causes minimal disruption of epitopes. Specimens fixed by freezing can subsequently be processed for
The author is at

the Laboratoire d'Analyse

Ultrastructurale, Bâtiment de Biologie, Université de Lausanne, $\mathrm{CH}-1015$ Lausanne, Switzerland. leaves us with a tough question: how much of the native biological structure remains? Cryoelectron microscopy makes it possible to keep water in the specimen but creates a different problem: water changes to ice upon cooling, and ice is very different to liquid water. As a consequence, the structure of most frozen specimens is more severely damaged by the growth of ice crystals than conventionally prepared specimens are damaged by dehydration. Figure 1 illustrates the fate of a poorly frozen cell. Considerable effort has been made to improve conditions of freezing by increasing the cooling speed, by adding cryoprotectants or by choosing well-behaved specimens. These efforts have resulted in a family of successful preparation methods such as freeze-fracture and freeze-substitution (Box 1 and Ref. 2). However, these methods are always accompanied by the threat of ice formation.

In the early 1980s, it was found, unexpectedly, that pure water can be vitrified - that is, it can be cooled so rapidly that it becomes immobilized before it has time to crystallize ${ }^{3,4}$. The old dream of cryobiologists became a reality and life could be suspended to allow the study of the inside of the cell. The discovery was soon turned into practical methods for electron microscopy ${ }^{5,6}$. Nowadays, biological specimens are studied best when fully hydrated in a thin vitrilied layer, and water has become the electron microscopist's best friend.

Unfortunately, vitrification only works well for biological samples that can be prepared in a thin layer of submicrometer thickness. For larger specimens, vitrification is much more difficult. Taking advantage of the natural

\section{BOX 1 - THE METHODS OF CRYOELECTRON MICROSCOPY}

(a) Dry specimen (in electron microscope)

Freeze-fracture:

The specimen is frozen, then fractured. A replica of the fractured surface is observed.

Freeze-drying:

The specimen is frozen, then lyophilized. The remaining dry material is stabilized for direct observation, or a replica is made.

Freeze-etching:

A combination of both freeze-fracture and freeze-drying.

Freeze-substitution:

The specimen is frozen, then the ice is dissolved and replaced at low temperature by an organic solvent (e.g. acetone). Embedding in plastic follows at low or normal temperature.

Cryo-immunocytochemistry:

The specimen is prepared in such a way as to obtain the best possible preservation of the immunogenicity for specific labelling. In general, the first step is a chemical fixation, followed by impregnation with a concentrated cryoprotectant (e.g. saturated sucrose solution). The specimen is frozen in order to obtain good cutting conditions for subsequent immunolabelling of the thawed sections.

(b) Hydrated specimen (in cryoelectron microscope)

Thin vitrified film (bare grid method, Adrian method...):

A thin layer $(\sim 1 \mu \mathrm{m})$ of cell suspension is vitrified by plunging in an efficient cryogen and observed directly in an electron microscope at temperatures below $-160^{\circ} \mathrm{C}$. This is a widely used, easy and reproducible preparation method.

Frozen sections:

The specimen is frozen, then cut into thin frozen sections that are observed in a cryoelectron microscope. The basic test for quality is whether the specimen is vitreous or not. When this is the case, the most faithful representation of the native sample is obtained. This is potentially the ideal preparation method but it is difficult and still not widely used.

\section{Staring at an icy problem}

The subtle art of preserving fine details and beauty in fully dehydrated biological specimens is a major achievement of 50 years of conventional electron microscopy, but it 
cryoprotective effect of many biological tissues and using efficient cooling systems, vitrification can frequently be achieved down to a depth of $10-20 \mu \mathrm{m}$.llt is then possible to prepare thin vitrified sections and to observe them directly by cryoelectron microscopy. Consequently, freezeetching or freeze-substitution, can also be used with the certainty that the starting material is free of damage by ice. However, it must be kept in mind that vitreous water is highly unstable, turning into ice as soon as the temperature rises above approximately $-100^{\circ} \mathrm{C}$, although the damage under these conditions is probably less severe than when ice forms during cooling ${ }^{7}$. It is unfortunate that $10-20 \mu \mathrm{m}$ of excellent preservation is not much, often not even enough to get out of the region that was damaged when the sample was taken from the organism.

\section{High-pressure freezing: improving cryopreservation}

One of the many surprising properties of water is that it increases in volume when it changes from a liquid to a solid. As most liquids cool, they become more ordered and they occupy less space (by analogy, when it is no longer possible to enter my children's room because of its disorder, I request they tidy it; the increase in order decreases the amount of space the children's belongings occupy). With water, it is just the contrary - the disordered liquid increases in volume when it becomes ordered ice. If the increase in volume is made more difficult by cooling the water at high pressure, growth of ice crystals is impaired. In the absence of crystal formation, the morphological preservation of biological samples is improved.

The principle of HPF is simple, but putting it into practice was more difficult ${ }^{8,9}$. It is now 25 years since the prototype of a high-pressure freezing machine was developed. Other appliances were built later, and now two commercial firms are marketing similar systems (Balzers; Leica; prices: -US\$200 000). In these machines, a pressure of -2000 bar is applied in some tens of milliseconds while a double jet of liquid nitrogen is blown on the $\sim 1 \mathrm{~mm}^{3}$ sample protected between thin $(\sim 0.1 \mathrm{~mm})$ aluminium plates. After less than one second, the jets stop, pressure is released and the cold specimen is ready for further processing.

How much improvement does HPF really bring? That EM is more art than science is perhaps illustrated by the fact that, 25 years after the method was first developed, the literature is rich in beautiful micrographs (Fig. 2; see Refs 10 and 11 for review), but very little is known for sure about its full potential. Of course, a good micrograph may speak for the method, but experience has shown repeatedly that the end product is even more indicative of the skill of the experimenter and the effort he put into the work. To determine the extent of the improvement that HPF can effect, we really need quantitative data and comparisons between specimens in which all the major parameters are known. In particular, it is important to know about the depth of vitrification, the exact nature of the specimen, its water content and the presence of cryoprotectant. Comparison between the results obtained by high- and nomalpressure freezing is meaningless unless at least these parameters are defined. Such quantitative analyses are still exceptional (U. Riehle, PhD Thesis, ETH, Zürich, 1968; Refs 12 and 13) and much remains to be done before we can access the true capabilities and limits of the method. Obtaining such quantitative data is not easy and some more facts concerning water and ice can help resolve the problems (see Ref. 14 for a review).

\section{Prevention of nucleation}

High pressure makes it difficult for ice crystals to grow. However, the crucial event in ice formation is not so much the growth of the crystal as the initial nucleation event. Crystal initiation requires a rare event that can be described as many water molecules 'finding', by chance, how to nucleate a crystal. It is because nucleation is so rare that vitrification is sometimes possible. When crystallization occurs, a large amount of energy is released in the specimen (sufficient, in principle, to rewarm the liquid by $80^{\circ} \mathrm{C}$ ) Obviously, this prevents further cooling until the liberated heat is withdrawn from the specimen. When freezing is performed under high pressure, the specimen is relatively large and not in direct contact with the cryogenic agent because it must be protected by a metal sheet. These conditions are not ideal for efficient

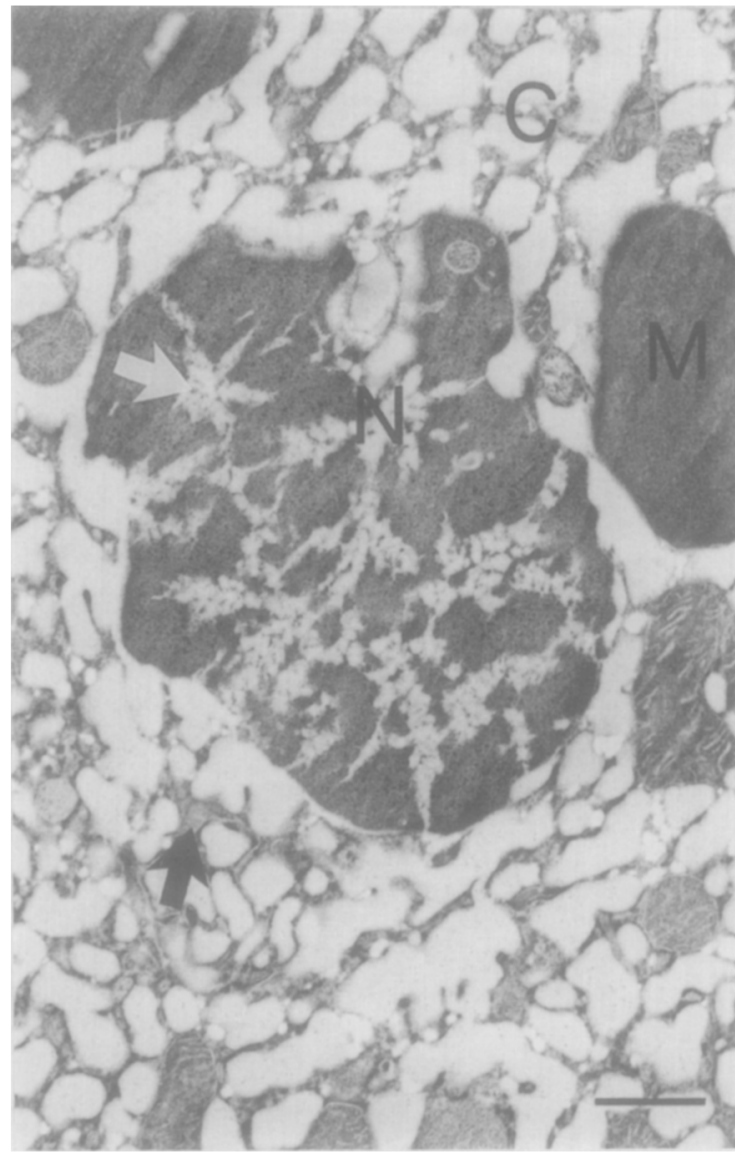

\section{FIGURE 1}

Damage due to freezing. A thin section of rat liver frozen by projection onto a cold metal block and cryosubstituted in a low temperature embedding resin K4M (Ref. 16). The section was made to a depth of $\sim 200 \mu \mathrm{m}$ into the specimen. The specimen has suffered severe damage due to freezing. During freezing, the material was segregated in two phases: condensed, dehydrated biological material (black arrows) and ice crystals. The latter, removed during freeze-substitution, leave only 'holes' (white arrows) in the meshwork of the dehydrated sample. When a similar sample is observed by cryoelectron microscopy with all its water still present, it can be demonstrated that there is not one ice crystal per hole but one, or a few, large, ramified crystals covering the whole volume of the cell. $\mathrm{C}$, cytoplasm; N, nucleus; $M$, mitochondria. Bar, $0.5 \mu \mathrm{m}$. (Micrograph by Dr M. L. von Schack.)

removal of the heat of crystallization.

There are some more subtle facets to HPF. Above a pressure of $2000 \mathrm{bar}$, the normal forms of ice are replaced by less usual forms such as ice II, ice III and ice IX, each having a density of $\sim 1.2$. It has been found recently that these types of ice are frequently formed under the typical conditions of HPF (Ref. 15) - with pernicious consequences for biological structures.

Finally, the sudden application of a pressure of 2000 bar is likely to change many things in a cell. Processes such 


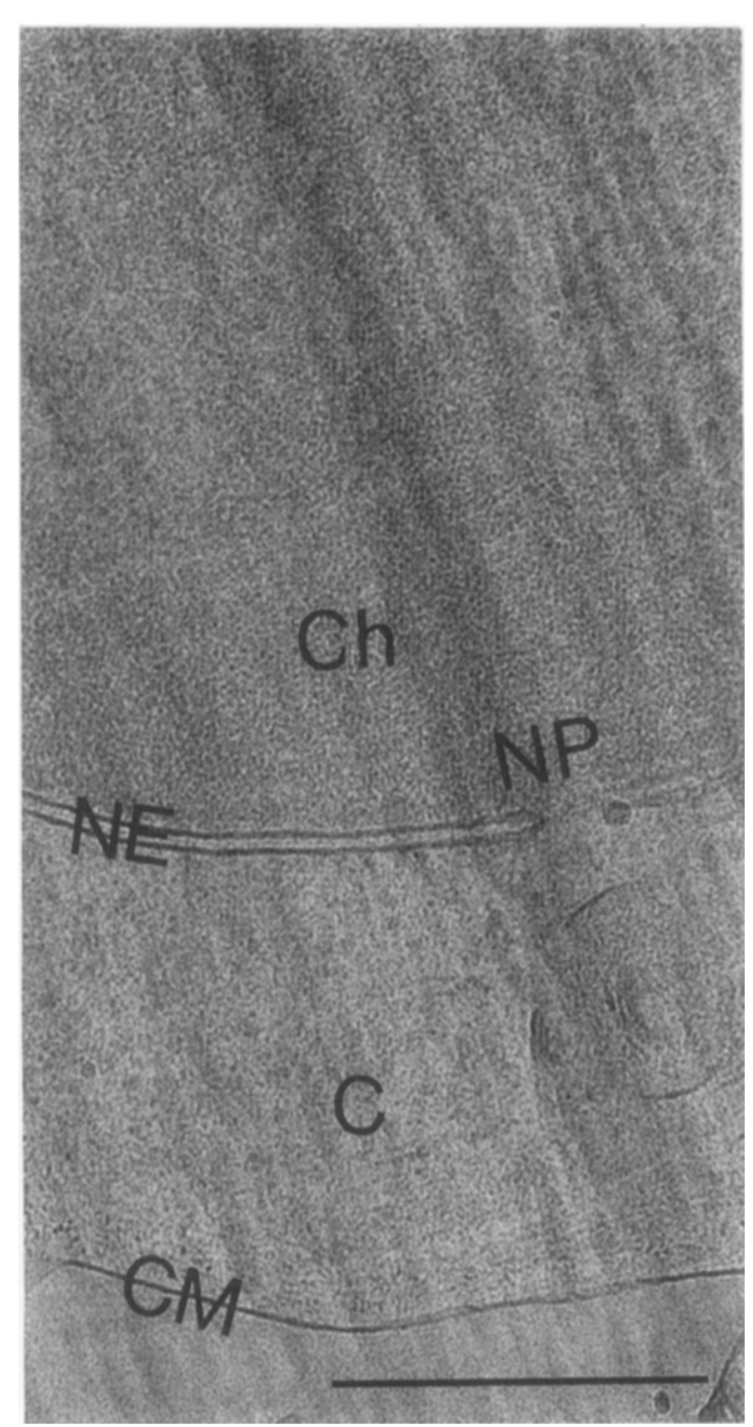

FIGURE 2

A well-preserved specimen. Thin vitreous section of a lymphocyte. A cell suspension was suspended in 15\% dextran and cooled under high pressure. The section was obtained by cryo-ultramicrotomy, to $\sim 100 \mu \mathrm{m}$ below the surface. The section, which is neither chemically fixed nor stained, was observed at a temperature of approximately $-170^{\circ} \mathrm{C}$. Electron diffraction confirms that the specimen is hydrated and that there are no ice crystals. The major components of the cell are visible. As compared to a conventionally prepared specimen, a major difference is that the condensed chromatin appears as a smooth 'sea of nucleosomes ${ }^{\prime 17}$. CM, cell membrane; $\mathrm{C}$, cytoplasm; $\mathrm{NE}$, nuclear envelope with nuclear pore (NP); Ch, condensed

chromatin. Bar, $0.2 \mu \mathrm{m}$. (Micrograph by N. Sartori.)

Acknowledgements

I thank N. Sartori Whether some of the changes are so for Fig. 2 and rapid as to cause important structural apologize to transformations under conditions of DrM. L. von Schack rapid freezing is still an open question, for the unflattering but the present results suggest that Fig. 1. these effects are not dramatic.

\section{A personal view of high-pressure freezing}

After studying all the information currently available on the process of HPF, I should like to offer the following thoughts on the technique:

- High-pressure freezing is a breakthrough. Using the technique, the depth of vitrification possible for most biological tissues has increased from $\sim 10 \mu \mathrm{m}$ to some $100 \mu \mathrm{m}$. Specimens that are free of damage due to ice formation can now be studied well outside the zone damaged when the sample was taken out of the organism. In practice, this changes the life of a cryoelectron microscopist. But...

- High pressure does not facilitate vitrification of pure water or dilute solutions. Extracellular fluid must be protected by an efficient cryoprotectant, such as sucrose, dextran or glycerol, at a concentration that, in our hands, is not less than $15 \%$. If this is not done, ice forms everywhere in the extracellular space, with generally unacceptable structural consequences for the cells.

- Certain operating conditions result in the formation of high-pressure forms of ice. This also has unacceptable morphological consequences. It is not yet clear how this is best avoided.

- Even in the presence of cryoprotectant and in the absence of highdensity ice, good freezing is still not sufficiently reproducible. There are further parameters that need to be characterized quantitatively and optimized; these relate both to the sample and to the apparatus.

In conclusion, the application of high pressure must be seen as a promising tool of cryoelectron microscopy, but cryoelectron microscopists must still learn how to get the best out of it. One thing they will not achieve, however, is 'suspended life' for themselves. Luyet can rest in peace*.

"Father Basile J. Luyet (1897-1974) was the first president of the Society for
Cryobiology. He was fascinated by the 'nature of life' and was concerned with the possible unethical use of cryobiology. His spirit is still present in the society and among many cryobiologists.

\section{References}

1 GOODSELL, D. A. (1991)Trends Biochem. Sci. 16, 203-206

2 STEINBRECHT, R. A. and ZIEROLD, K., eds (1987) Cryotechniques in Biological Electron Microscopy, Springer

3 MAYER, E. and BRÜGGELLER, P. (1980) Nature 288, 569-571

4 DUBOCHET, J. and MCDOWALL, A. W. (1981) ). Microsc. 124, RP3-RP4

5 ADRIAN, M., DUBOCHET, I., LEPAULT, I. and MCDOWALL, A. W. (1984) Nature 308, 32-36

6 DUBOCHET, I. et al. (1988) Q. Rev. Biophys. 21, 129-228

7 DUBOCHET, J., RICHTER, K., ROY, H-V. and MCDOWALL, A. W. (1991) Scanning Electron Microsc. (Suppl. 5),

S11-S16

8 MOOR, H. and RIEHLE, U. (1968) in Electron Microscopy (Vol. 2) (Bocciarelli, S., ed.), pp. 33-34, Proc. 4th Eur. Reg. Conf. Electron Microsc., Rome

9 MOOR, H. (1987) in Cryotechniques in Biological Electron Microscopy (Steinbrecht, R. A. and Zierold, K., eds) pp. 175-191, Springer

10 DAHL, R. and STAEHLIN, L. A. (1989) J. Electron Microsc. Tech. 13, 165-174

11 STUDER, D., MICHEL, M. and MÜLLER, M. (1989) Scanning Electron Microsc. 3, 253-269

12 SARTORI, N., RICHTER, K. and DUBOCHET, J. (1993) J. Microsc. 172, 55-61

13 STUDER, D., MICHEL, M., WOHLVEND, M., HUNZIKER, E. B. and BUSCHMANN, M. J. Microsc. (in press)

14 FRANKS, F., ed. (1982) in Properties of Aqueous Solutions at Subzero Temperatures (Vol. 7), pp. 215-338, Plenum Press

15 RICHTER, K. (1994) Uitramicroscopy 53, 237-249

16 VON SCHACK, M. L. and FAKAN, S. (1993) Micron 24, 507-519

17 MCDOWALL, A. W., SMITH, J. M. and DUBOCHET, J. (1986) EMBO /. 5 , 1395-1402

\section{MISCELLANEA}

The MISCELLANEA section of trends in CELL BIOLOGY may include techniques articles, reports of conferences, reviews of books, and other occasional features. Techniques articles outline the application of new and developing techniques to problems in cell biology. Meeting reports highlight recent advances and new ideas aired at conferences of particular relevance to cell biologists. Book reviews provide a focus for discussion of a range of topics in cell biology, as well as opinion on new publications. These articles are usually commissioned by the editorial team, but trends in CELL BIOLOCY welcomes suggestions for future contributions to MISCELLANEA. 